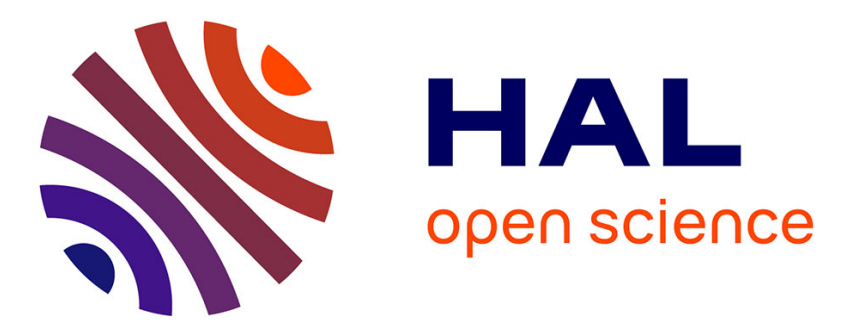

\title{
Timing recovery scheme for multi-antenna receiver in presence of Doppler shift
}

\author{
Goulven Eynard, Christophe Laot
}

\section{To cite this version:}

Goulven Eynard, Christophe Laot. Timing recovery scheme for multi-antenna receiver in presence of Doppler shift. ICT 2018: International Conference on Telecommunication, Jun 2018, Saint-Malo, France. 10.1109/ICT.2018.8464943 . hal-01814199

\section{HAL Id: hal-01814199 \\ https://hal.science/hal-01814199}

Submitted on 11 Jun 2021

HAL is a multi-disciplinary open access archive for the deposit and dissemination of scientific research documents, whether they are published or not. The documents may come from teaching and research institutions in France or abroad, or from public or private research centers.
L'archive ouverte pluridisciplinaire HAL, est destinée au dépôt et à la diffusion de documents scientifiques de niveau recherche, publiés ou non, émanant des établissements d'enseignement et de recherche français ou étrangers, des laboratoires publics ou privés. 


\title{
Timing recovery scheme for multi-antenna receiver in presence of Doppler shift
}

\author{
Goulven Eynard ${ }^{1}$ and Christophe Laot $^{2}$ \\ ${ }^{1}$ DGA Maitrise de l'information, Route de Laille, 35131 Bruz \\ Email: goulven.eynard@intradef.gouv.fr \\ 2 IMT Atlantique, LabSTICC, UBL, 29238 Brest cedex \\ Email: christophe.laot@imt-atlantique.fr
}

\begin{abstract}
This paper proposes a timing recovery tracking scheme for single-input multiple-output (SIMO) communications derived from the maximum likelihood criterion for an all-digital single carrier receiver. The timing recovery scheme proposed is able to take advantage of the spatial diversity at the receiver side, especially when a common time scaling is present over the multiple antennas. This phenomenon appears when the sampling rate at the receiver is different of its value at the transmitter or in broadband communications with moving platforms. The SIMO timing recovery tracking scheme is applied to single carrier phase-coherent modulations over records of underwater acoustic communications. The proposed method allows improving the performance of the estimation or equalization of SIMO channels in comparison with conventional timing recovery SISO schemes applied independently on each antenna.
\end{abstract}

Index Terms-Synchronization, timing recovery, underwater communications, spatial diversity, clock drift, Doppler shift, single input multiple output (SIMO), digital locked loop.

\section{INTRODUCTION}

From the theory of digital communications, an optimal coherent receiver requires that synchronization (timing and carrier recovery) and equalization are performed jointly [1], [2]. In practice, it is difficult to estimate jointly the whole parameters of a coherent receiver especially when the parameters are timevarying. Independent and sub-optimum estimators are often preferred where timing recovery and coarse carrier recovery are performed before the equalization or channel estimation.

In many conventional phase-coherent receivers, the optimum time sampling and carrier phase are obtained using a matched filter to a pilot sequence which is introduced in the transmitted data slot. This data aided (DA) approach allows estimation of time sampling and carrier phase but it assumes that the receiver clock is accorded to the transmitter clock and there are no carrier phase offset. In many practical applications, these hypothesis are wrong and it requires the using of banks of filters jointly matched to the pilot sequence, to the carrier phase offset and to the clock offset. In function of the chosen accuracy of the parameters to be estimate, a matched filter bank can lead to a large computational complexity. Moreover, if an estimated parameter changes on the duration of the communication, it must be periodically estimate leading to short slot and a loss of spectral efficiency due to the insertion of periodic pilot sequences. Another solution consists to track the time-varying parameters by an adaptive estimation based on digital locked loops.

In this paper, we assume a time-varying clock drift between the receiver and transmitter. This phenomenon can be due to a lack of accuracy of the embedded oscillators or to the Doppler effect induced by a relative movement between the transmitter and the receiver. The Doppler effect is particularly sensible in underwater acoustic communications where the small velocity of the acoustic waves results in a time-scaling or a Doppler shift of the received waveform [3], [4]. To compensate the clock drift or Doppler shift, a coarse estimation and compensation scheme is often required [3], [5]. However, when the channels are time and frequency selective, the instantaneous signal to noise ratio (SNR) can be low and the estimation of Doppler shift can fail. In a multiple antenna context, the spatial diversity is a solution to improve the Doppler shift estimation. Unfortunately, the presence of different time delays propagation on each receiver antenna prohibits the use of coherent tracking estimation. In this specific case, a timing recovery scheme able to provide an independent time sampling on each receiver antenna and estimate a common Doppler shift using the spatial diversity that will be of prime interest to improve the performance of the receiver. That is the objective of this paper.

The timing recovery scheme proposed is able to take advantage of the spatial diversity of SIMO communications improving the receiver performance in comparison with conventional SISO timing recovery scheme applied independently on each antenna. The main novelty concerns the possibility to estimate jointly the common Doppler (or sampling rate) shift using the multiple antennas and compensate independently the propagation delays on each antenna. Theoretical developments justify the proposed approach. To implement this timing recovery scheme, we have retained a digital locked loop which is computationally efficient compared to a bank of correlators and allows to track time variation of the parameters to be estimate. A non data aided (NDA) timing recovery tracking loop is considered to avoid joint time and phase synchronization required in the data-aided mode. Although, the theoretical developments assume the knowledge of the matched filter at the receiver side, that is not the case in a practical context, it is shown that the proposed SIMO timing recovery tracking scheme is efficient with real sea experiments. 


\section{TIME-SCALING CHANNEL MODEL AND OPTIMUM RECEIVER ARCHITECTURE}

\section{A. Time-scaling due to Doppler in wireless transmissions}

In a general case of communication with moving platforms, Doppler effect is known to introduce a time-scaling factor $a=(1-v / c)$ where $v$ (resp. $c$ ) is the relative speed between the platforms (resp. the speed of sound in the sea) [3], [5]. Considering a receiver with $N_{R}$ antennas, the received signal at the antenna $i=1, \ldots, N_{R}$ can be modeled as a time-scaling of the transmitted signal waveform:

$$
r_{i}(t)=s\left(a t-\tau_{i}\right)+w_{i}(t)
$$

where $\tau_{i}$ is the propagation channel delay at the antenna $i, w_{i}(t)$ is an additive white Gaussian noise (AWGN) with spectral power density $N_{0} / 2$, and $s(t)$ is the transmitted waveform of the signal:

$$
s(t)=\Re e\left\{\sum_{n=-\infty}^{+\infty} d_{n} g(t-n T) e^{j\left(2 \pi f_{c} t+\psi\right)}\right\}
$$

where $f_{c}$ is the carrier frequency, $\psi$ is a constant carrier phase uncertainty, $T$ is the symbol-time duration and $\left\{d_{n}\right\}$ is the transmitted $M$-PSK data symbol vector. $g(t)$ is the received pulse-shape which can encompass a frequency selective channel.

In the following part, the optimum receiver architecture in the ML sense is derived, taking into account the time-scaling factor due to the Doppler.

\section{B. Optimal receiver in the $M L$ sense}

Assuming all channel parameters known or perfectly estimated at the receiver side, the ML criterion can be expressed as:

$$
\hat{\mathbf{d}}=\arg \max _{\tilde{\mathbf{d}}} L\left(\left\{r_{i}(t)\right\} \mid \tilde{\mathbf{d}}\right),
$$

where $L\left(\left\{r_{i}(t)\right\} \mid \tilde{\mathbf{d}}\right)$ is the likelihood function with $i=$ $1, \ldots, N_{R}$, the vector $\tilde{\mathbf{d}}=\left\{\tilde{d}_{n}\right\}$ represents the set of the trial symbols to be estimated, and $\hat{\mathbf{d}}=\left\{\hat{d}_{n}\right\}$ is the vector of the decided symbols, which satisfies the ML criteria. Assuming the AWGN noise signals $w_{i}(t)$ mutually independent on each antenna $i$, maximizing the ML cost function for an $M$-PSK transmission is equivalent to maximizing [6], [7]:

$$
J\left(\left\{r_{i}(t)\right\} \mid \tilde{\mathbf{d}}\right)=\exp \left\{\frac{2}{N_{0}} \sum_{i=1}^{N_{R}} \int_{-\infty}^{+\infty} r_{i}(t) \tilde{x}_{i}^{*}(t) d t\right\},
$$

where the superscript * stands for the complex conjugate operator and $\tilde{x}_{i}(t)$ is a trial of the received waveform expressed as:

$$
\tilde{x}_{i}(t)=\Re e\left\{\sum_{n=-\infty}^{+\infty} \tilde{d}_{n} g\left(a t-\tau_{i}-n T\right) e^{j\left(2 \pi f_{c}\left(a t-\tau_{i}\right)+\psi\right)}\right\} .
$$

From the ML criterion (3), the optimum decision vector $\hat{\mathbf{d}}$ is obtained by maximizing the summation of correlation integrals given by $R_{\tilde{\mathbf{d}}}$ and defined as:

$$
R_{\tilde{\mathbf{d}}}=\sum_{i=1}^{N_{R}} \int_{-\infty}^{+\infty} r_{i}(t) \tilde{x}_{i}^{*}(t) d t
$$

Maximizing the ML cost function is equivalent to maximize (6) which simplifies to :

$$
R_{\tilde{\mathbf{d}}}=\sum_{i=1}^{N_{R}} \sum_{n=-\infty}^{+\infty} \Re e\left\{\tilde{d}_{n}^{*} p\left(\left(\tau_{i}+n T\right) / a\right)\right\},
$$

where $p_{i}(t)$ is the matched filter output after demodulation and $\tau_{i, n}=\left(\tau_{i}+n T\right) / a$ is the optimum sampling time which can be expressed as:

$$
\tau_{i, n}=n T+n \frac{v / c}{1-v / c} T+\frac{1}{1-v / c} \tau_{i}
$$

The optimum sampling time is expressed as the clock time $n T$ corrected by a time-variant term which encompasses the common Doppler shift and the propagation delay of the considered antenna. A practical implementation of an all-digital SIMO receiver is plotted on Fig.1.

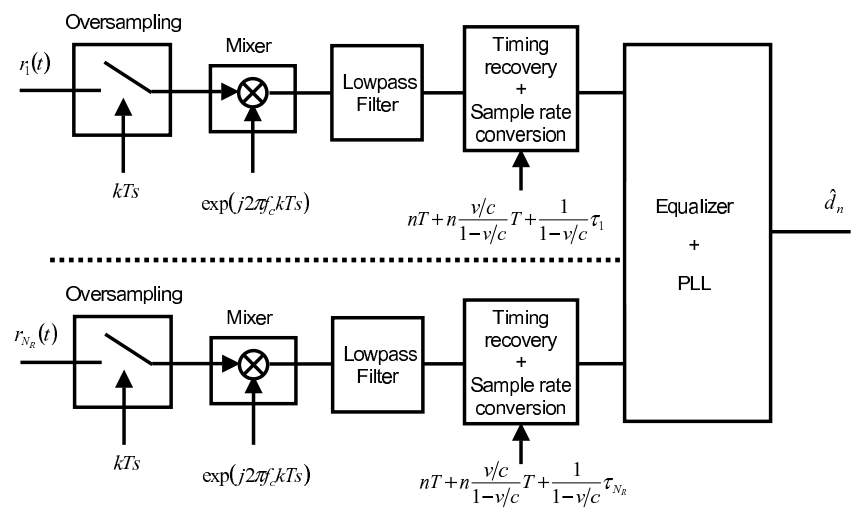

Fig. 1. Practical implementation of an all-digital SIMO receiver.

Since the relative velocity of the platforms $v$ and the propagation velocity $c$ can change on a continuous-time communications, a timing recovery scheme must be used in order to track the optimum sampling time. The following section presents a NDA timing recovery tracking scheme dedicated to SIMO receivers.

\section{JOINT CLOCK DRIFT AND CLOCK DELAY COMPENSATION SCHEME}

In this section, we show theoretically that the common Doppler shift must be estimate and compensate jointly using all the antennas whereas the propagation delays different on each antenna must be compensate independently.

A tracking scheme is defined by its TED (timing error detector) [7], which can be obtained by the derivation of the cost function (4). The ML criterion (7) can be optimized in 
a data aided (DA) approach considering the insertion of pilot symbols. Nevertheless, a DA timing recovery scheme requires an accurate carrier-phase recovery before or jointly to the timing recovery increasing the complexity of the synchronization process. For this reason, non data aided (NDA) timing recovery schemes are often used in spite of a performance loss between DA and NDA approaches [8], [9].

A NDA timing recovery tracking scheme can be obtained by computing the expectation of the likelihood function (4) over all transmitted symbols. The resulting NDA likelihood function is defined as:

$$
\bar{L}(\tilde{\boldsymbol{\tau}})=E_{\tilde{\mathbf{d}}}\left\{L\left(\left\{r_{i}(t)\right\} \mid \tilde{\mathbf{d}}, \tilde{\boldsymbol{\tau}}\right)\right\}
$$

Writing the ML likelihood function leads to:

$$
L\left(\left\{r_{i}(t)\right\} \mid \tilde{\mathbf{d}}, \tilde{\boldsymbol{\tau}}\right) \propto \exp \left\{\frac{2}{N_{0}} \sum_{i=1}^{N_{R}} \sum_{n=-\infty}^{+\infty} \Re e\left\{\tilde{d}_{n}^{*} p\left(\tilde{\tau}_{i, n}\right)\right\}\right.
$$

and the estimated sampling instant $\tilde{\tau}_{i, n}(8)$ can be expressed as:

$$
\tilde{\tau}_{i, n}=n T+\tilde{\mathcal{D}}_{n}+\tilde{\mathcal{D}}_{i, n}
$$

where $\tilde{\mathcal{D}}_{n}$ is the common clock drift and $\tilde{\mathcal{D}}_{i, n}$ with $i=$ $1, \ldots, N_{R}$ are the different propagation delays on each antenna $i$. Computations details of the extraction of the TED from the ML cost function are given in the Appendix. The resulting optimum sampling instant on each antenna $i$ can be expressed by the following relation:

$$
\hat{\tau}_{i, n+1}=\hat{\tau}_{i, n}+T-K_{1} \sum_{j=1}^{N_{R}} u_{j, n}-K_{2} u_{i, n}
$$

where $K_{1}$ and $K_{2}$ are two suitable constant step-sizes and $u_{i, n}$ is the resulting output of the early-late SISO TED which can be expressed as:

$$
u_{i, n}=\Re e\left\{\left(p_{i}\left(\hat{\tau}_{i, n}-\frac{T}{2}\right)-p_{i}\left(\hat{\tau}_{i, n}+\frac{T}{2}\right)\right) p_{i}^{*}\left(\hat{\tau}_{i, n}\right)\right\}
$$

Notice that the sampling instant (12) on the antenna $i$ is update from two errors terms. The first term is a sum calculated from the TEDs of all the antennas allowing to track the common clock drift. The second term depends only of the TED of the antenna $i$ allowing the compensation of different static delays between each antenna. From our knowledge, such timing recovery using this spatial diversity scheme has not been presented in the literature.

The early-late SISO TED (13) can be replaced by the Gardner SISO TED [10] which is often used in literature [7]. The Gardner SISO TED can be expressed as:

$$
u_{i, n}=\Re e\left\{\left(p_{i}\left(\hat{\tau}_{i, n}-T\right)-p_{i}\left(\hat{\tau}_{i, n}\right)\right) p_{i}^{*}\left(\hat{\tau}_{i}(n)-\frac{T}{2}\right)\right\}
$$

Notice that the early-late TED or the Gardner TED could be replaced by any TED based on decision directed or dataaided criterion. In this case, a joint phase compensation will be required.

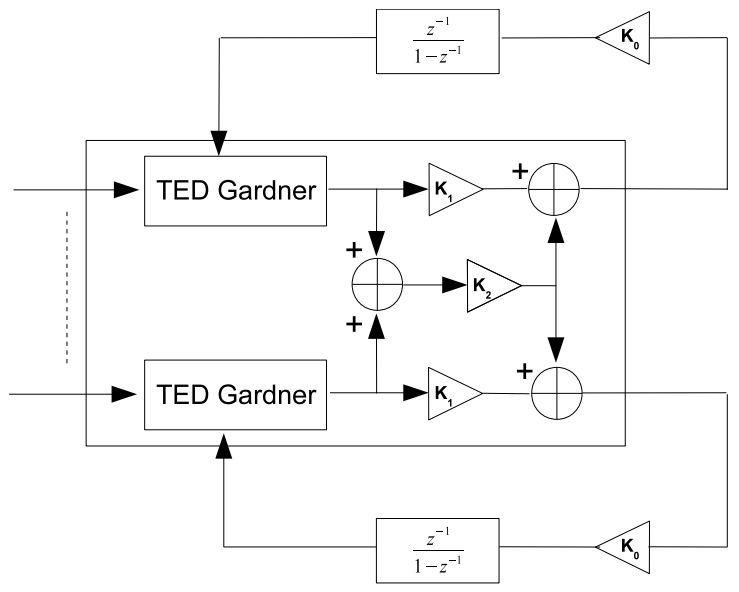

Fig. 2. SIMO timing recovery tracking scheme.

The SIMO timing recovery scheme is depicted in Fig.2. Its implementation is based on a low complexity digital locked loop (DLL). The setting of the parameters $K_{1}$ and $K_{2}$ depends of the lock-in and tracking ranges [2], [11]. The behavior study and theoretical performance in term of error variance are out of the scope of this paper.

It can be noticed that the SIMO closed loop estimation scheme iteratively drives $N_{R}+1$ error signals to zero, which are related to $N_{R}$ independent sampling delays and one clock drift estimation error. Thus, the ML SIMO closed loop tracking scheme can be seen as a generalization of two SIMO tracking scheme solutions:

- The SIMO clock drift compensation scheme which iteratively drives to zero the sum of the SISO TED on all antennas. It can be obtained by taking $K_{2}=0$ on (12) and Fig.2. This solution is the ML solution when there is no sampling delays between each receiver antennas.

- The SIMO clock delay compensation scheme, denoted NDA SISO, which iteratively drives to zero each TED independently. It can be obtained by taking $K_{1}=0$ on (12) and Fig.2. This solution is the ML solution when there is no common clock drift on each antennas.

The proposed generalization of these two TED is denoted NDA SIMO. In the result section, the NDA SIMO will be compare with $N_{R}$ NDA SISO timing recovery scheme performing independently on each antenna.

\section{EXPERIMENTAL RESULTS}

Experimental sea trials were carried in bay of Brest, France [12]. The transmitter and the receiver were placed on a ship and on a quay, respectively. A QPSK transmission with carrier frequency $35 \mathrm{kHz}$ was used during the trials. The receiver has $N_{R}=4$ antennas (hydrophones) deployed linearly and spaced from approximately $25 \mathrm{~cm}$. On this received signal record, the distance between the transmitter and the receiver is about 1200 $\mathrm{m}$, the depth is $20 \mathrm{~m}$, the symbol rate is $1 / T=5000 \mathrm{symb} / \mathrm{s}$ and the oversampling factor is $F_{s}=28$. 

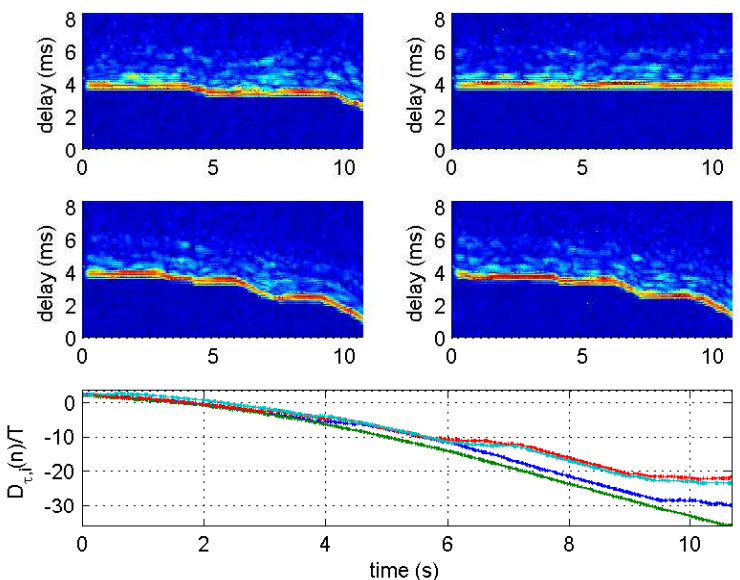

Fig. 3. Top: estimation of $N_{R}=4$ time-varying CIR over a record of underwater acoustic SIMO communication versus time. Bottom: evolution of the time sampling on the $N_{R}=4$ antennas using $N_{R}=4$ independent NDA SISO TED recovery schemes versus time.
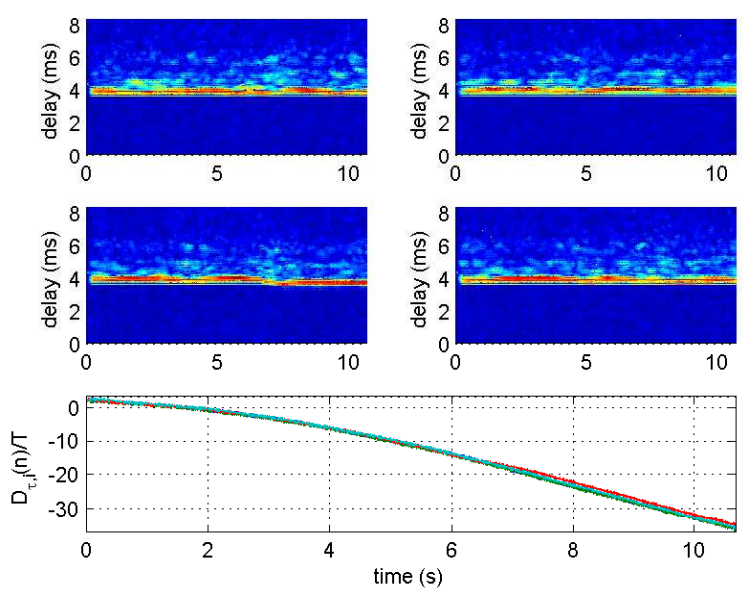

Fig. 4. Top: estimation of $N_{R}=4$ time-varying CIR over a record of underwater acoustic transmission versus time. Bottom: evolution of the time sampling on the $N_{R}=4$ antennas using the NDA SIMO TED recovery scheme versus time.

The tracking behavior of the proposed timing recovery scheme is evaluated on a transmission signal records of duration 10 seconds. The time evolution of the estimated time-varying sampling instant denoted $D_{\tau, i}(n)=\hat{D}_{n}+\hat{D}_{i, n}$ from (20) is analyzed and plotted in the bottom of Fig.3 and Fig.4. The estimation of the channel impulse response (CIR) on each antenna is performed after the time sampling using $N_{R}=$ 4 conventional and independent NDA SISO TED recovery schemes (Fig.3) or the proposed NDA SIMO TED recovery scheme (Fig.4). The CIR estimation plots give the evolution of the multi-paths delays versus time.

In the first configuration of the timing recovery tracking scheme, the conventional and independent NDA SISO TEDs are used. Fig. 3 depicts the tracking process on a duration of $10 \mathrm{~s}$. On this time duration, many cycle slips appear, introducing a delay of 40 symbol periods for a relative velocity greater than $v=1 \mathrm{~m} / \mathrm{s}$ at the end of the acceleration period. At the top of the Fig. 3 is also plotted the CIR estimation on each antenna using a least mean square (LMS) data aided algorithm. It can be seen in Fig. 3 that the adaptive LMS-DA algorithm forces the estimation to shift the path delays versus the cycle slips introduced in the timing recovery process. In a practical experimentation, the LMS-DA will be generally replaced by a decision directed LMS algorithm which is insensible to cycle slips of the time recovery scheme, resulting to the suppression of symbols or insertion of fictive symbols, engendering a cutoff in the communication.

In the Fig.4, the proposed NDA SIMO TED is used on the same transmission signal record. On a time duration of $10 \mathrm{~s}$, few cycle slips are observed. The channel estimation process appears not to be impacted by the Doppler shift. The NDA SIMO TED improves the robustness of the tracking scheme. Consequently, the proposed method able to reduce drastically the cycle slips allows improving of the communication reliability. For time-selective channels, the improvement will be function to the number $N_{R}$ of antennas. Notice that for the experiments at sea, the matched filter $p_{i}(t)$ was unknown and replaced by a low-pass filter. That differs on the theoretical developments provided in this paper. Although this approach is sub-optimum, the experimental results shown the proposed NDA SIMO TED is robust and efficient over time and frequency selective channels.

\section{CONCLUSIONS}

This paper presents a timing recovery loop using the antenna diversity at the receiver side to improve the estimation and the tracking of a time-varying clock drift due to the Doppler shift. The mathematical demonstrations, issue from the ML criterion, prove that if a common clock drift exists between antennas, it is profitable to be considered in the optimization process. The resulting timing error detector can be seen as a spatial extension of conventional error detectors. The proposed timing recovery loop allows a smart use of spatial diversity. This scheme has been tested on real underwater communications in a shallowwater context allowing reliable and robust communications over a long time duration. Firstly developed for underwater acoustics communications, it could be advantageously used in digital communication systems where oscillators are inaccurate or in satellite communications where Doppler shift needs to be also considered. For future work, it would be interesting to consider the using of the CIR estimations to approximate the matched filters allowing to focus the energy of multi-paths before the time recovery scheme.

\section{APPENDIX}

The optimization algorithm proposed here consists in an iterative maximization of a cost function expressed as:

$$
\bar{L}(\tilde{\boldsymbol{\tau}})=E_{\tilde{\mathbf{d}}}\left\{\exp \left\{\frac{2}{N_{0}} \sum_{i=1}^{N_{R}} \sum_{n=1}^{N} \Re e\left\{\tilde{d}_{n}^{*} p_{i}\left(\tilde{\tau}_{i, n}\right)\right\}\right\} \mid \tilde{\mathbf{d}}, \tilde{\boldsymbol{\tau}}\right\}
$$


Assuming that $\left\{d_{n}\right\}$ are i.i.d (independant and identically distributed) $M$-PSK data symbols, the cost function can be exprimed :

$$
\bar{L}(\tilde{\boldsymbol{\tau}}) \propto \prod_{n=1}^{N} \prod_{i=1}^{N_{R}} \frac{1}{M} \sum_{\left\{d_{n}\right\}} \exp \left\{\frac{2}{N_{0}} \Re e\left\{d_{n}^{*} p_{i}\left(\tilde{\tau}_{i, n}\right)\right\}\right\}
$$

Considering a QPSK, $M=4$, and $d_{n}=(1 / \sqrt{2})\left(a_{n}+j b_{n}\right)$ with $a_{n}, b_{n} \in\{ \pm 1\}$, produces:

$$
\bar{L}(\tilde{\boldsymbol{\tau}}) \propto \frac{1}{4} \prod_{n=1}^{N} \prod_{i=1}^{N_{R}} \sum_{\left\{a_{n}, b_{n}\right\}} \exp \left\{\frac{\sqrt{2}}{N_{0}}\left\{a_{n} p c_{n, i}+b_{n} p s_{n, i}\right\}\right\}
$$

where $p c_{n, i}=\Re e\left\{p_{i}\left(\tilde{\tau}_{i, n}\right)\right\}$ and $p s_{n, i}=\Im m\left\{p_{i}\left(\tilde{\tau}_{i, n}\right)\right\}$. Then, using the cosh function, :

$$
\bar{L}(\tilde{\boldsymbol{\tau}}) \propto \prod_{n=1}^{N} \prod_{i=1}^{N_{R}} \cosh \left\{\frac{\sqrt{2}}{N_{0}} p c_{n, i}\right\} \cosh \left\{\frac{\sqrt{2}}{N_{0}} p s_{n, i}\right\}
$$

Taking the logarithm of $\bar{L}(\tilde{\boldsymbol{\tau}})$, the equivalent cost function to maximize is given as:

$$
\bar{\Lambda}(\tilde{\boldsymbol{\tau}})=\sum_{n=1}^{N} \sum_{i=1}^{N_{R}}\left[\log \cosh \left\{\frac{p c_{n, i}}{\frac{N_{0}}{\sqrt{2}}}\right\}+\log \cosh \left\{\frac{p s_{n, i}}{\frac{N_{0}}{\sqrt{2}}}\right\}\right]
$$

where the sampling time trial is given by $\tilde{\tau}_{i, n}=n T+\tilde{\mathcal{D}}_{n}+$ $\tilde{\mathcal{D}}_{i, n}$ whereas the optimum sampling time maximizing the ML criterion will be denoted:

$$
\hat{\tau}_{i, n}=n T+\hat{\mathcal{D}}_{n}+\hat{\mathcal{D}}_{i, n}
$$

To solve the optimization problem, we resort to a recursive procedure given by:

$$
\hat{\tau}_{j, n+1}=\hat{\tau}_{j, n}+T-\left.K_{1} \frac{\partial \bar{l}(\hat{\tau})}{\partial \hat{\mathcal{D}}}\right|_{\hat{\mathcal{D}}=\hat{\mathcal{D}}_{n}}-\left.K_{2} \frac{\partial \bar{l}(\hat{\tau})}{\partial \hat{\mathcal{D}}_{j}}\right|_{\hat{\mathcal{D}}_{j}=\hat{\mathcal{D}}_{j, n}}
$$

where $\bar{l}(\hat{\tau})$ is expressed as:

$\bar{l}(\hat{\tau})=\sum_{i=1}^{N_{R}} \log \cosh \left\{\frac{\Re e\left\{p_{i}\left(\hat{\tau}_{i}\right)\right\}}{\frac{N_{0}}{\sqrt{2}}}\right\}+\log \cosh \left\{\frac{\Im m\left\{p_{i}\left(\hat{\tau}_{i}\right)\right\}}{\frac{N_{0}}{\sqrt{2}}}\right\}$

and $K_{1}$ and $K_{2}$ are two step-size factor. Using the derivate of $\log \cosh (x)$ equal to $\tanh (x)$, the following expression is obtained:

$$
\frac{\partial \bar{l}(\hat{\tau})}{\partial \hat{\mathcal{D}}}=\sum_{i=1}^{N_{R}} \Re e\left\{\overline{p_{i}^{*}\left(\hat{\tau}_{i}\right)} \frac{\partial p_{i}\left(\hat{\tau}_{i}\right)}{\partial \hat{\mathcal{D}}}\right\}
$$

where $\overline{p_{i}\left(\hat{\tau}_{i}\right)}$ is a soft decision of the matched filter output:

$\overline{p_{i}\left(\hat{\tau}_{i}\right)}=\tanh \left\{\frac{\sqrt{2}}{N_{0}} \Re e\left\{p_{i}\left(\hat{\tau}_{i}\right)\right\}\right\}+j \tanh \left\{\frac{\sqrt{2}}{N_{0}} \Im m\left\{p_{i}\left(\hat{\tau}_{i}\right)\right\}\right\}$

Using the derivate approximation [7]:

$$
\frac{\partial p_{i}\left(\hat{\tau}_{i}\right)}{\partial \hat{\mathcal{D}}} \approx \frac{1}{T}\left(p_{i}\left(\hat{\tau}_{i, n}-\frac{T}{2}\right)-p_{i}\left(\hat{\tau}_{i, n}+\frac{T}{2}\right)\right)
$$

leads to the expression of the TED on the antenna $i$ on time $\hat{\tau}_{i}(n)$ :

$$
u_{i}(n)=\frac{1}{T} \Re e\left(\left(p_{i}\left(\hat{\tau}_{i}(n)-\frac{T}{2}\right)-p_{i}\left(\hat{\tau}_{i, n}+\frac{T}{2}\right)\right) \overline{p_{i}^{*}\left(\hat{\tau}_{i, n}\right)}\right)
$$

Considering the derivate about $\hat{\mathcal{D}}_{j}$ :

$$
\frac{\partial \bar{l}(\hat{\tau})}{\partial \hat{\mathcal{D}}_{j}}=\Re e\left\{\overline{p_{j}^{*}\left(\hat{\tau}_{j}\right)} \frac{\partial p_{j}\left(\hat{\tau}_{j}\right)}{\partial \hat{\mathcal{D}}_{j}}\right\}
$$

then, substituting (23) and (27) in (21), resolves the optimization problem and produces the following equation:

$$
\hat{\tau}_{j, n+1}=\hat{\tau}_{j, n}+T-K_{1} \sum_{i=1}^{N_{R}} u_{i, n}-K_{2} u_{j, n}
$$

Note that when $x$ is low, the approximation $\tanh (x) \approx x$ is valid, the soft decision can be expressed as:

$$
\overline{p_{i}^{*}\left(\hat{\tau}_{i}\right)} \approx \frac{\sqrt{2}}{N_{0}} p_{i}^{*}\left(\hat{\tau}_{i}\right)
$$

giving the error signal of the well-known early-late detector (ELD) on the antenna $i$ on time $\hat{\tau}_{i}(n)$ :

$$
u_{i}(n) \propto \Re e\left(\left(p_{i}\left(\hat{\tau}_{i, n}-\frac{T}{2}\right)-p_{i}\left(\hat{\tau}_{i, n}+\frac{T}{2}\right)\right) p_{i}^{*}\left(\hat{\tau}_{i}(n)\right)\right)
$$

\section{REFERENCES}

[1] J. Proakis, Digital Communications. 3nd edition, Mc Graw Hill, 1995.

[2] H. Meyr, M. Moeneclaey, and S. Fechtel, Digital Communication Receivers: Synchronization, Channel Estimation, and Signal Processing. New York: Wiley, 1998.

[3] B. Sharif, J. Neasham, O. Hinton, and A. E. Adams, "A computationaly efficient doppler compensation system for underwater acoustic communications," IEEE J. Oceanic Eng., vol. OE-25, pp. 52-61, Jan. 2000.

[4] S. Daoud and A. Ghayeb, "Using resampling to combat doppler scaling in uwa channels with single-carrier modulation and frequency-domain equalization," IEEE Transactions on Vehicular Technology, vol. 65, pp. 1261-1270, Mar. 2016.

[5] L. Freitag, M. Johnson, and D. Frye, "High-rate acoustic communications for ocean observatories-performance testing over a $3000 \mathrm{~m}$ vertical path," Proc. of OCEANS'00, pp. 1443-1448, Sept. 2000.

[6] F. Gardner, Demodulator Reference Recovery Techniques Suited for Digital Implementation, ser. ESTEC Contract No. 6847/86/NL/DG. European Space Agency, 1988.

[7] U. Mengali and A. D'Andrea, Synchronization Techniques for Digital Receivers. Springer, 1997.

[8] R. Chellappa, S. Theodoridis, M. Ghogo, P. Ciblat, and A. Swami, Academic Press Library in Signal Processing: Communications and Radar Signal Processing, Chapter 2: Synchronization. Elsevier, 2014.

[9] I. Nasr, L. N. Atallah, S. Cherif, and B. Geller, "Time synchronization in iot networks: Case of a wireless body area network," in International Symposium on Signal, Image, Video and Communications (ISIVC), Nov 2016, pp. 297-301.

[10] F. Gardner, "A bpsk/qpsk timing-error detector for sampled receiver," IEEE Trans. Commun., vol. COM-34, pp. 423-429, May. 1986.

[11] G. Eynad and C. Laot, "Extended linear phase detector characteristic of a software pll," in International Symposium on Communications, Control and Signal Processing (ISCCSP), March 2008.

[12] — "Blind doppler compensation scheme for single carrier digital underwater communications," in IEEE Oceans'08, September 2008. 